\title{
Neoareolar Wise Pattern Reduction in Patients Requiring Central Partial Mastectomy
}

\author{
Janice Kang, MD, Debra Wechter, MD, and Janie Grumley, MD \\ General Surgery, Virginia Mason Medical Center, Seattle, WA
}

\begin{abstract}
Background. Oncoplastic surgery integrates breast cancer resection with tissue transfer techniques to preserve breast cosmesis. With the novel methods developed in the oncoplastic movement, we are capable of performing larger resections with clear margins while minimizing the cosmetic deformity caused by more traditional surgical techniques. Central and retroareolar breast cancers continue to be a cosmetic challenge because removal of the central portion of the breast can be particularly deforming. Methods. An inferior pedicle Wise pattern reduction mammoplasty is performed. The inferior pedicle is deepithelized except for a circular disc of skin superiorly, which will become the neoareola. The breast cancer is removed along with the nipple-areolar complex (NAC). Part of the NAC may be preserved if tumor resection can be performed with sufficient margins. A standard reduction is performed, removing more tissue as indicated by
\end{abstract}

cosmetic need. The reduction is completed in standard fashion using the neoareola as a replacement for the previously removed NAC.

Results. The video demonstrates the feasibility of this technique, highlighting both preservation of breast shape and contour, and creation of a substitute for the NAC.

Discussion. Complex reduction techniques have been reported in the literature as feasible options for breast conservation surgery. The neoareolar Wise pattern reduction mammoplasty offers an option that allows for oncologically sound cancer surgery while simultaneously preserving cosmesis.

CONFLICT OF INTEREST The authors declare no conflict of interest.
Electronic supplementary material The online version of this article (doi:10.1245/s10434-013-3149-5) contains supplementary material, which is available to authorized users.

(C) Society of Surgical Oncology 2013

First Received: 11 April 2013;

Published Online: 22 August 2013

J. Grumley, MD

e-mail: janie.grumley@vmmc.org 